\title{
Crescimento do meloeiro irrigado com águas de diferentes salinidades ${ }^{1}$
}

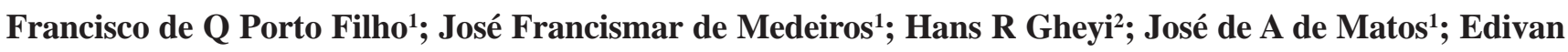 \\ R de Souza ${ }^{1}$; Eduardo R de Sousa Neto ${ }^{1}$ \\ ${ }^{1}$ UFERSA-Dep ${ }^{\text {to }}$ Ciências Ambientais, Mossoró-RN; ${ }^{2}$ UFCG-CCT-DEAg, Campina Grande-PB; porto@ufersa.edu.br
}

\begin{abstract}
RESUMO
Dois experimentos consecutivos foram realizados na mesma área, em Mossoró-RN, com o objetivo de estudar características de crescimento da cultura do melão submetida a diferentes níveis de salinidade da água de irrigação. Utilizou-se um delineamento experimental em blocos casualizados com quatro repetições, cujos tratamentos foram quatro níveis de salinidade da água $\left(\mathrm{T}_{1}=0,6 \mathrm{dS} \mathrm{\textrm {m } ^ { - 1 }}\right.$; $\mathrm{T}_{2}=1,9 \mathrm{dS} \mathrm{m}^{-1} ; \mathrm{T}_{3}=3,2 \mathrm{dS} \mathrm{m}^{-1}$ e $\mathrm{T}_{4}=4,5 \mathrm{dS} \mathrm{m}^{-1}$ ). Os níveis de salinidade da água de irrigação afetaram negativamente o índice de área foliar, fitomassa seca da parte aérea, área foliar específica e razão de área foliar da parte aérea, inibindo o crescimento. A taxa de crescimento absoluto da parte aérea foi afetada pelo nível de salinidade da água de irrigação, enquanto para a taxa de crescimento relativo e a taxa de assimilação líquida da parte aérea não foi observado efeito dos sais ao longo do ciclo do meloeiro.
\end{abstract}

Palavras-chave: $\mathrm{C}$ cumis melo L, qualidade da água, taxa de assimilação.

\begin{abstract}
Growth of melon plants irrigated with waters of different salinities

Two successive trials were carried out in a field at Mossoró, $\mathrm{RN}$, Brazil, with the objective of studying growth characteristics of melon crop under different irrigation levels of water salinity. The experimental design was a completely randomized blocks with four replications, with four water salinity levels as treatments $\left(\mathrm{T}_{1}=0.6 \mathrm{dS}\right.$ $\mathrm{m}^{-1} ; \mathrm{T}_{2}=1.9 \mathrm{dS} \mathrm{m}^{-1} ; \mathrm{t}_{3}=3.2 \mathrm{dS} \mathrm{m}^{-1}$ and $\left.\mathrm{T}_{4}=4.5 \mathrm{dS} \mathrm{m}^{-1}\right)$. Growth was inhibited by the negative effect of water salinity levels on leaf area index, shoot dry mass, specific leaf area, and shoot leaf area ratio. The influence of irrigation of the water salinity level along melon cycle was observed for shoot absolute growth rate, but not for relative growth rate and net assimilation rate of shoot.
\end{abstract}

Keywords: Cucumis melo L, water quality, assimilation rate.

(Recebido para publicação em 28 de julho de 2005; aceito em 1 de setembro de 2006)

$\mathrm{O}$ Brasil produz $349.498 \mathrm{t}^{\mathrm{ano}^{-1}}$ de melão, dos quais $330.720 \mathrm{t}^{\mathrm{ano}} \mathrm{o}^{-1}$ são produzidos na Região Nordeste. O Estado do Rio Grande do Norte é o maior produtor, respondendo por $55 \%$ do melão brasileiro (IBGE, 2003). Na região do Pólo Agrícola Mossoró/Açu, neste Estado, a maior demanda por água para irrigação tem condicionado à utilização de águas com salinidade mais elevada. Segundo Medeiros (1992), Martins (1993), Oliveira \& Maia (1998), embora a maioria das fontes de água apresente boa qualidade, existem águas de qualidade inferior nessa região que podem ser utilizadas na irrigação.

A salinização pode causar efeitos negativos nas plantas que diferem entre espécies e cultivares. A tolerância das culturas aos sais é afetada por vários fatores, incluindo-se aí o estágio de desenvolvimento, a duração da exposição, as condições ambientais, as propriedades do solo, o tipo e a intensidade do manejo (Maas, 1990). O meloeiro apre- senta grande variação no nível de tolerância à salinidade, variando tanto entre cultivares quanto com as condições ambientais e de manejo (Pizarro, 1990; Francois \& Maas, 1993). Nerson \& Paris (1984), estudando quatro cultivares de melão, observaram que a seleção para a tolerância à salinidade pode ser realizada a partir do início do desenvolvimento vegetativo da cultura, pois a redução na área foliar e na matéria seca das plantas jovens se equipara às reduções na produção promovidas pela salinidade. Nesta cultura, quando se aumenta a concentração salina da água de irrigação, diminui uma série de características da planta, como altura, peso fresco de folhas, ramos e raízes, peso seco, comprimento e diâmetro de entrenós e área foliar (Anastasio et al., 1987; Porto filho et al., 2001).

Segundo Awad \& Castro (1983), a velocidade de crescimento, a disposição, a área foliar e o número de folhas são responsáveis pela maior ou menor co- bertura da superfície do solo e, conseqüentemente, pela eficiência na interceptação da energia luminosa e no seu aproveitamento. Informações sobre a fisiologia da planta são fundamentais para o desenvolvimento de modelos de simulação e para a compreensão das interações entre planta e ambiente. Alguns índices fisiológicos, como o índice de área foliar (IAF) e taxa de crescimento da cultura (TCC), dentre outros, podem ser comparados na tentativa de explicar as diferenças na produção econômica de diferentes cultivares ou de uma mesma cultivar submetida a diferentes tratamentos (Machado et al., 1982). Na folha ocorrem as trocas gasosas executadas pelos vegetais, efetuando-se a fotossíntese e a transpiração, o que torna a área foliar uma característica muito utilizada em análise de crescimento (vigor) de plantas. O IAF, a TCC e o fator de cobertura são os meios mais acessíveis e precisos para avaliar o crescimento e contribuem em processos fi-

${ }^{1}$ Parte da tese de doutorado do $1^{\circ}$ autor, apresentada na Universidade Federal de Campina Grande. Trabalho financiado pelo Programa Avança Brasil, convênio Embrapa/UFERSA e pelo Projeto Melhoria do Rendimento e da Qualidade de Frutos Produzidos no Estado do RN, convênio FINEP/Fundação Guimarães Duque/UFERSA. 
siológicos e no comportamento vegetal (Benincasa, 1988).

Este trabalho teve como objetivo estudar características de crescimento da cultura do melão submetida a diferentes níveis de salinidade da água de irrigação.

\section{MATERIAL E MÉTODOS}

A pesquisa foi composta de uma seqüência de dois experimentos, conduzidos em 2001 e 2002, com o meloeiro (Cucumis melo L.), var. inodorus, cv. AF646, que apresenta frutos com casca de coloração amarela, no município de Mossoró-RN, num Latossolo Vermelho Eutrófico argissólico, textura média (Embrapa, 1999).

Nos experimentos aplicaram-se águas de irrigação, com quatro níveis de salinidade $\left(S_{1}=0,6, S_{2}=1,9, S_{3}=3,2\right.$ e $\mathrm{S}_{4}=4,5 \mathrm{dS} \mathrm{m} \mathrm{m}^{-1}$ ). Na Tabela 1 está a composição química das quatro águas utilizadas.

Os quatro tratamentos foram dispostos em blocos inteiramente casualizados, com quatro repetições, em 16 parcelas de $36 \mathrm{~m}^{2}(6,00 \times 6,00 \mathrm{~m})$. Cada parcela foi composta por três fileiras de planta no espaçamento entre fileiras de 2,00 m, e na fileira, de forma alternada, espaçamento de 0,38 e $0,12 \mathrm{~m}$ tendo uma planta por cova, resultando numa população de plantio de 20.000 plantas $\mathrm{ha}^{-1}$. O experimento II foi uma repetição do I, utilizando-se, exatamente o mesmo local, tratamentos e delineamento experimental.

A área experimental foi irrigada por gotejamento, através de quatro sistemas de irrigação independentes. As lâminas de irrigação foram determinadas a partir de estimativas da evapotranspiração da cultura utilizando-se metodologia do Kc dual proposto pela FAO (Allen et al., 1998) e ajustadas com base no monitoramento da umidade do solo com o uso de tensiômetros em duas repetições do tratamento $\mathrm{T}_{1}$, adotando-se uma fração de lixiviação de 0,10 . O Kc basal adotado para as fases inicial, intermediária e final correspondeu a $0,15,1,00$ e 0,70 , respectivamente. Os tensiômetros foram instalados nas profundidades de 0,15; 0,30 e 0,45 m numa distância média de $0,09 \mathrm{~m}$ da planta e num raio médio de $0,22 \mathrm{~m}$ do gotejador, na região central da parcela experimental. Diariamente, o potencial mátrico da água do solo (Ym), na profundidade de $0,15 \mathrm{~m}$, servia para indicar se no momento antes da irrigação a lâmina e a freqüência de irrigação adotada atendiam ao valor considerado crítico para o melão (-30 kPa). Tanto os instrumentos a $0,15 \mathrm{~m}$ como nas demais profundidades, serviram para quantificar a água armazenada no solo e, ao se calcular a lâmina de irrigação pela ETc, sabia-se se o solo era capaz de comportála, indicando se estava de acordo com a necessidade hídrica da planta. No experimento I, as lâminas de irrigação aplicadas nos períodos de 0 a 30, 31 a 50 e 51 a 70 dias após semeadura (DAS) foram, respectivamente, de 108, 136 e 101 $\mathrm{mm}$. No período entre um experimento e outro (264 dias), a precipitação pluviométrica foi de $1.228,00 \mathrm{~mm}$ e foram aplicados $381 \mathrm{~mm}$ de irrigação complementar em cultura intercalar de milho, usando-se a água $\mathrm{S}_{3}$. No experimento II, as lâminas de irrigação aplicadas nos períodos de 0 a 30, 31 a 50 e 51 a 68 DAS foram, respectivamente, de 95, 132 e $85 \mathrm{~mm}$.

Os valores médios de temperatura média (Tmed), máxima (Tmax) e mínima (Tmin), umidade relativa (UR), velocidade do vento a $10 \mathrm{~m}(\mathrm{~V})$, insolação (I) e ETo para os experimentos I e II foram de $28,9,35,0$ e $24,2{ }^{\circ} \mathrm{C}, 65,5 \%$, $5,5 \mathrm{~m} \mathrm{~s}^{-1}, 9,1 \mathrm{~h} \mathrm{e} 6,6 \mathrm{~mm} \mathrm{~d}^{-1}$ e de 28,4 , 35,1 e $23,2{ }^{\circ} \mathrm{C}, 62,8 \%, 6,0 \mathrm{~m} \mathrm{~s}^{-1}, 10,4 \mathrm{~h}$ e $7,1 \mathrm{~mm} \mathrm{~d}^{-1}$, respectivamente.

Em ambos os experimentos, as adubações foram realizadas de acordo com a análise de solo da área experimental, atendendo-se às exigências nutricionais da cultura, conforme Vivancos (1996) e distribuídas ao longo do ciclo, em conformidade com as curvas de absorção apresentadas por Lima (2001). A cultura foi mantida sempre no limpo, através de capinas manuais com uso de enxada. O controle das principais pragas e doenças comuns na região foi preventivo, através do uso de defensivos agrícolas.

O crescimento da cultura foi avaliado através da coleta de uma planta por parcela, aos 14, 21, 29, 36, 49 e 65 DAS no experimento I, e aos 16, 23, 30, 37, 44, 51, 58 e 64 DAS no experimento II, a partir de plantas localizadas nas duas fileiras externas de cada parcela. As características avaliadas foram: número de folhas (NF), área do limbo foliar (AF), fitomassa seca da parte aérea (FSPA), subdividida em fitomassas secas de ramos (FSR), folhas (FSF) e frutos (FSFr). A área foliar foi determinada através de um integrador de área, marca LI-COR, modelo LI-3100, enquanto a fitomassa foi obtida por secagem em estufa de circulação de ar forçada a $65{ }^{\circ} \mathrm{C}$ e posterior pesagem em balança com precisão $0,01 \mathrm{~g}$.

A partir de FSPA, FSF e da AF obtidas nos diversos tempos de coletas e da área de ocupação do solo por cada planta (A), nos experimentos I e II, foram calculadas a área foliar específica (AFE), razão de área foliar da parte aérea (RAFPA), índice de área foliar (IAF), taxa de crescimento absoluto da parte aérea (TCAPA), taxa de crescimento relativo da parte aérea (TCRPA) e taxa de assimilação líquida da parte aérea (TALPA).

Os índices AFE, RAFPA e IAF foram determinados a partir de valores absolutos de cada coleta nos experimentos I e II, de FSPA, FSF, AF e A, através das equações $\mathrm{AFE}=\mathrm{AF} / \mathrm{FSF}\left(\mathrm{cm}^{2} \mathrm{~g}^{-1}\right)$; $\mathrm{RAFPA}=\mathrm{AF} / \mathrm{FSPA}\left(\mathrm{cm}^{2} \mathrm{~g}^{-1}\right)$ e IAF $=\mathrm{AF} /$ A $\left(\mathrm{m}^{2} \mathrm{~m}^{-2}\right)$, propostas por Benincasa (1988).

Com os dados das características AF, FSPA, AFE, RAFPA e IAF, procedeuse à análise de variância, com ajuste de equações de regressão. A TCAPA foi determinada derivando-se a equação ajustada para FSPA em relação ao tempo (t). A TCRPA e a TALPA foram estimadas através da relação entre a equação de TCAPA e as equações de melhor ajuste que fornecem, respectivamente, os valores instantâneos de FSPA e AF, conforme Radford (1967), Richards (1969), Negreiros (1995) e Rocha (2001), por meio das equações TCAPA= $d(\mathrm{FSPA}) / d \mathrm{t}\left(\mathrm{g} \mathrm{planta}^{-1} \mathrm{dia}^{-1}\right)$, TCRPA $=$ TCAPA/FSPA $\left(\mathrm{g} \mathrm{g}^{-1} \mathrm{dia}^{-1}\right)$ e TALPA= TCAPA/AF $\left(\mathrm{g} \mathrm{m}^{-2} \mathrm{dia}^{-1}\right)$.

As características de crescimento das plantas foram analisadas por experimento, utilizando-se parcela subdividida para os tratamentos repetidos no tempo (DAS) (Lima, 1996) e procedeuse à análise de regressão, a fim de se 


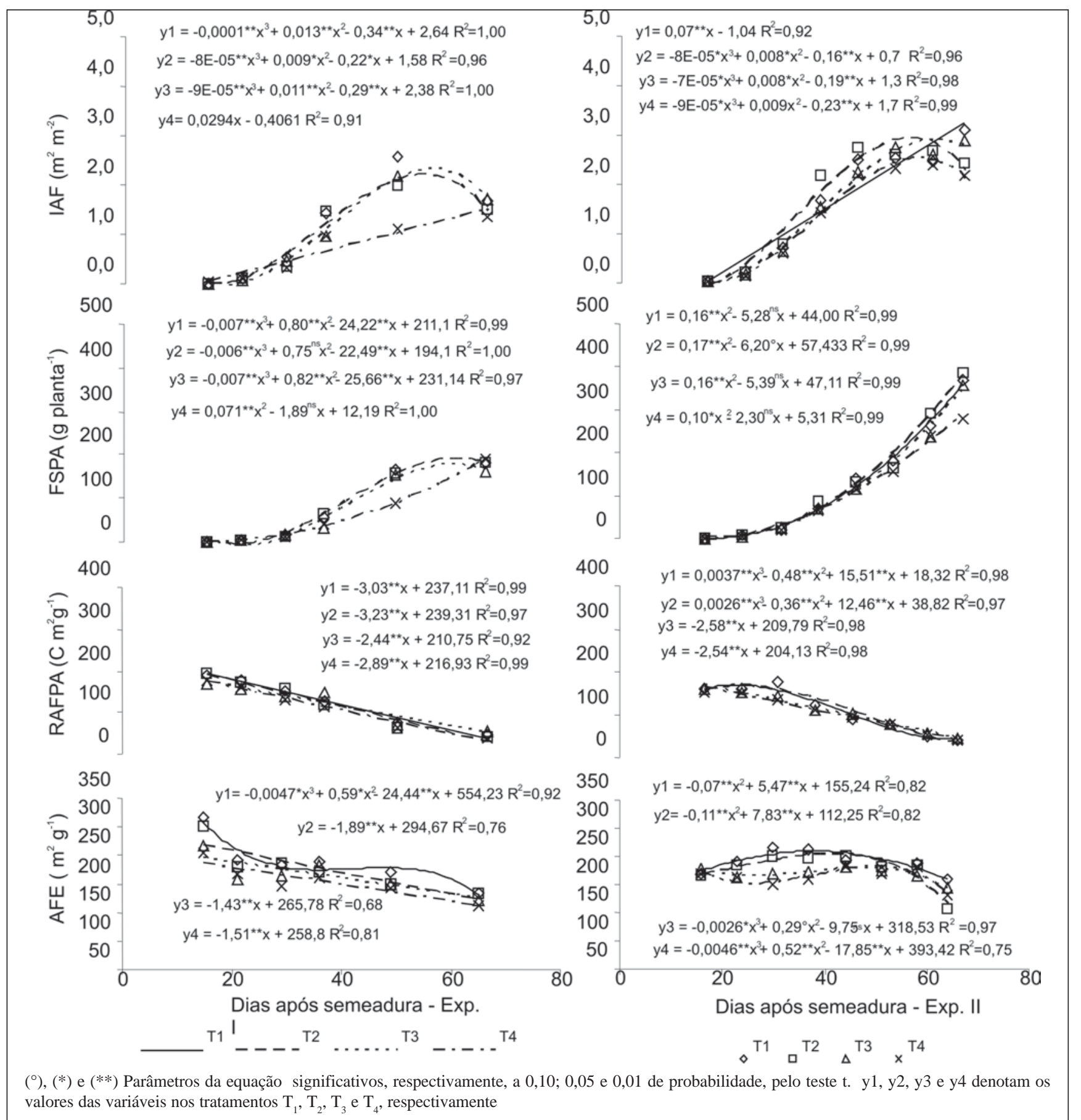

Figura 1. Índice de área foliar (IAF), fitomassa seca da parte aérea (FSPA), razão de área foliar da parte aérea (RAFPA) e área foliar específica (AFE) do melão irrigado submetido a diferentes níveis de salinidade nos Experimentos (Exp.) I e II. Mossoró-RN, 2001 e 2002.

estabelecer relações entre as características de crescimento e a idade da planta para cada tratamento, com o emprego de polinômios ortogonais (Gomes, 1987).

\section{RESULTADOS E DISCUSSÃO}

No experimento I, pela análise de variância de índice de área foliar (IAF), fitomassa seca da parte aérea (FSPA), área foliar específica (AFE) e razão de área foliar da parte aérea (RAFPA), os efeitos de tratamento foram significativos a 0,05 de probabilidade para IAF e não significativos para as outras três características; no fator tempo (DAS), a significância foi a 0,01 de probabilidade, em todas as características. O aumento da salinidade da água de irrigação diminuiu linearmente as caracterís- ticas avaliadas, como expressam as equações: $\mathrm{IAF}\left(\mathrm{m}^{2} \mathrm{~m}^{-2}\right)=-0,094 * * \mathrm{CEa}$ $+1,116\left(\mathrm{R}^{2}=0,87\right)$; FSPA $\left(\mathrm{g}\right.$ planta $\left.^{-1}\right)=-$ $4,041 * \mathrm{CEa}+74,386\left(\mathrm{R}^{2}=0,93\right) ; \mathrm{AFE}$ $\left(\mathrm{cm}^{2} \mathrm{~g}^{-1}\right)=-8,705^{*} \mathrm{CEa}+243,25\left(\mathrm{R}^{2}=\right.$ $1,00)$; RAFPA $\left(\mathrm{cm}^{2} \mathrm{~g}^{-1}\right)=-3,495^{*} \mathrm{CEa}$ $+131,06\left(\mathrm{R}^{2}=0,86\right)$. Na Tabela 2 encontram-se os valores médios de índice de área foliar (IAF), fitomassa seca da parte aérea (FSPA), área foliar específi- 


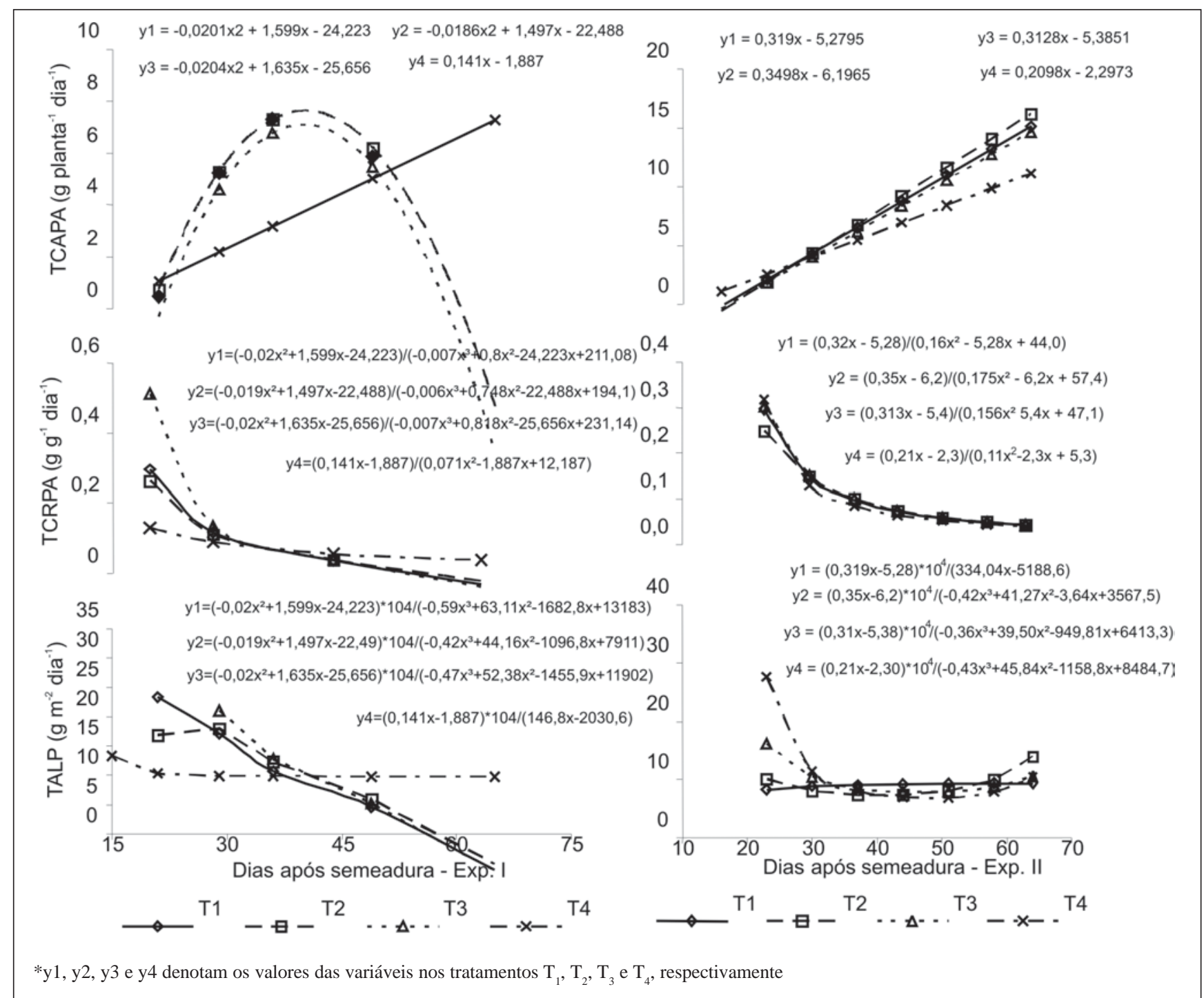

Figura 2. Taxa de crescimento absoluto da parte aérea (TCAPA), taxa de crescimento relativo da parte aérea (TCRPA) e taxa de assimilação líquida da parte aérea (TALPA) do melão irrigado submetido a diferentes níveis de salinidade nos Experimentos (Exp.) I e II. Mossoró-RN, 2001 e 2002.

ca (AFE) e razão de área foliar da parte aérea (RAFPA) nos experimentos I e II.

No desdobramento de DAS dentro de cada nível de salinidade (tratamento) para o IAF, a resposta foi cúbica para os três tratamentos menos salinos e linear para o tratamento mais salino (Figura 1); as equações indicaram valores de IAF máximo aos 54, 54 e 56 DAS e maior taxa de crescimento aos $36,35 \mathrm{e}$ 37 DAS, respectivamente, nos tratamentos $\mathrm{T}_{1}, \mathrm{~T}_{2}$ e $\mathrm{T}_{3}$. Em $\mathrm{T}_{4}$, na última coleta (65 DAS), obteve-se o maior IAF, o que indica retardamento no crescimento devido, provavelmente, ao estresse salino. Este índice continuou crescendo no final do ciclo devido ao estresse hídrico imposto pela diminuição da lâmina de irrigação no final do ciclo para a maturação dos frutos ter sido igual ao determinado para $\mathrm{T}_{1}$, afetando menos $\mathrm{T}_{4}$, que se encontrava com IAF menor. Resultados semelhantes a estes foram encontrados por Oliveira (1995) para o meloeiro híbrido Valenciano Amarelo (Gold-Mine), cujo IAF foi maior aos 51 e 70 DAS, respectivamente, para condições sem e com estresse hídrico. Costa (1999), utilizando este mesmo híbrido, evidenciou que o estresse salino no período crítico da cultura até os 36 DAS afeta significativamente o restante do ciclo.

Para FSPA, de forma semelhante ao IAF, a resposta foi cúbica aos tratamentos $\mathrm{T}_{1}, \mathrm{~T}_{2}$ e $\mathrm{T}_{3}$ e quadrática com concavidade para cima, de grande abertura em $\mathrm{T}_{4}$ (Figura 1). Pelas equações, verificam-se valores de FSPA máximo aos 59, 60 e 59 DAS, respectivamente, nos tratamentos $\mathrm{T}_{1}, \mathrm{~T}_{2}$ e $\mathrm{T}_{3}$, e maior taxa de crescimento em todos a 40 DAS. A defasagem entre as épocas para se atingir os valores máximos e de maior taxa de crescimento da FSPA, em relação ao IAF, indica a estreita relação existente entre a área foliar e a atividade fotossiteticamente ativa que tem, como resposta, o acúmulo de matéria seca (Benincasa, 1988).

A AFE teve ao longo do ciclo resposta cúbica em $\mathrm{T}_{1}$ e linear decrescente em $\mathrm{T}_{2}, \mathrm{~T}_{3}$ e $\mathrm{T}_{4}$ (Figura 1). Em $\mathrm{T}_{1}$, a AFE estimada através da equação cúbica 
Tabela 1. Composição química das águas com quatro níveis de salinidade (S) utilizadas no trabalho. Mossoró-RN, 2001 e 2002.

\begin{tabular}{|c|c|c|c|c|c|c|c|c|c|c|c|}
\hline Água & $\mathrm{pH}$ & CE & $\mathrm{Ca}$ & Mg & $\mathrm{Na}$ & $\mathrm{K}$ & $\mathrm{CO}_{3}$ & $\mathrm{HCO}_{3}$ & $\mathrm{Cl}$ & $\mathrm{SO}^{4}$ & RAS \\
\hline & (dS m & & \multicolumn{8}{|c|}{$\left(\mathrm{mmolc} \mathrm{L}^{-1}\right)$} & $\left(\mathrm{mmol}_{\mathrm{c}} \mathrm{L}^{-1}\right)^{1 / 2}$ \\
\hline${ }^{1 S_{1}}$ & 7,4 & 0,6 & 1,83 & 1,79 & 2,96 & 0,54 & 0,74 & 3,13 & 1,67 & Aus & 2,20 \\
\hline${ }^{2} \mathrm{~S}_{2}$ & 7,3 & 1,9 & 6,90 & 2,68 & 8,64 & 0,37 & 0,42 & 4,63 & 12,23 & Pres & 3,95 \\
\hline${ }^{3} \mathrm{~S}_{3}$ & 7,1 & 3,2 & 13,43 & 3,83 & 15,96 & 0,14 & 0,00 & 6,56 & 25,86 & Pres & 5,43 \\
\hline${ }^{4} \mathrm{~S}_{4}$ & 7,1 & 4,5 & 13,43 & 3,83 & 23,86 & 0,14 & 0,00 & 6,56 & 33,76 & Pres & 8,12 \\
\hline
\end{tabular}

1água de poço do aquíífero Arenito Açu, prof. de $1000 \mathrm{~m} ;{ }^{2}$ água obtida pela mistura de $\mathrm{S}_{1}$ com $\mathrm{S}_{3}$; ${ }^{3}$ água de poço do aqüúfero Calcário Jandaíra, prof. de $80 \mathrm{~m} ;{ }^{4}$ preparada pela adição de $\mathrm{NaCl}$ a água $\mathrm{S}_{3}$,

ajustada (Figura 1), apresentou valores de $306,260,231,225,230$ e $185 \mathrm{~cm}^{2} \mathrm{~g}^{-1}$, respectivamente, para 15, 21, 29, 36, 49 e 65 DAS, sendo as determinações aos 36 e 49 DAS os pontos de mínimo e máximo, respectivamente, e maior taxa de crescimento aos 42 DAS. Embora se tenha verificado pequena elevação na AFE aos 49 DAS, houve uma tendência de diminuição desta característica com a idade das plantas, conforme verificado também por Rocha (2001) para o feijão de corda consorciado com o milho. Embora essas oscilações em AFE, resultantes da taxa de crescimento individual das folhas, possam ocorrer, elas são bastante complexas e difíceis de serem explicadas (Benincasa, 1988). Portanto, ressalta-se que a equação linear decrescente: $\mathrm{AFE}=-2,004 * *$ DAS + 310,06 $\left(R^{2}=0,74\right)$, com maior significância de $F(p<0,01)$ e ligeira diminuição do coeficiente de determinação $\left(\mathrm{R}^{2}\right)$ pode representar o comportamento da AFE para $T_{1}$, de forma idêntica ao ocorrido em $\mathrm{T}_{2}, \mathrm{~T}_{3}$ e $\mathrm{T}_{4}$.

Para RAFPA, obteve-se efeito linear decrescente com o tempo nos quatro tratamentos. Esta característica, por relacionar a área foliar (AF) com toda FSPA acumulada, reflete a matéria seca produzida pelas folhas através da fotossíntese e distribuída para os outros constituintes da parte aérea; também, tende a apresentar menores oscilações de valores ao longo do tempo do que a AFE, que é determinada utilizando-se apenas a fitomassa seca da folha (FSF). A diminuição da RAFPA com o tempo parece ser ontogênica, indicando que, gradativamente, uma quantidade menor de assimilados é destinada às folhas (Negreiros, 1995 e Nóbrega et al., 2001).

Quando se determinou a TCAPA, mediante a derivada das equações ajus- tadas para a FSPA, verificaram-se equações de comportamento quadrático nos três primeiros níveis salinos $\left(\mathrm{T}_{1}, \mathrm{~T}_{2} \mathrm{e}_{3}\right)$ e linear no maior nível $\left(\mathrm{T}_{4}\right)$. Isto significa que na planta irrigada ao longo do ciclo, com níveis salinos até $3,2 \mathrm{dS} \mathrm{m}^{-1}$, a TCAPA cresceu até atingir um ponto máximo a 40 DAS (floração/ frutificação), diminuindo ao final do ciclo. Quando se analisa o tratamento mais salino, percebe-se que a planta teve crescimento retardado, ou seja, continuou crescendo até o final do ciclo, com um coeficiente angular da equação linear de 0,141 , o que pode ser constatado na Figura 2.

Ao se analisar a TCRPA (Figura 2), que representa todo o crescimento resultante da produção de material suficiente para atender às necessidades metabólicas do material já existente e, ainda, para armazenar e/ou construir novo material estrutural (Benincasa, 1988), verificou-se ter havido decréscimo dessa taxa ao longo do ciclo, com equações de comportamento quadrático para os quatro tratamentos. No entanto, o crescimento foi maior, inicialmente, nos três primeiros tratamentos, quando comparado com o nível mais salino, que variou pouco durante o ciclo da cultura. Oliveira (1995) verificou o mesmo comportamento de elevação e queda nas TCAPA para um híbrido de melão Valenciano Amarelo (Gold-Mine) cultivado sob condições sem e com estresse hídrico, com valores máximos aproximados aos, respectivamente, 40 e 36 DAS e, também, decréscimo na TCRPA ao longo do ciclo do melão, obtendo maiores valores iniciais e menores reduções no tratamento sem estresse. Por fim, ao se observar a TALPA, constatou-se que os tratamentos propiciaram maior ganho assimilatório no início, decrescendo posteriormente, diferente do tratamento mais salino, que não variou durante o ciclo da cultura.

De modo geral, a taxa de crescimento das plantas foi afetada pela salinidade. Para os tratamentos com salinidade inferior a $\mathrm{T}_{4}\left(\mathrm{~T}_{1}\right.$ a $\left.\mathrm{T}_{3}\right)$, a TCAPA cresceu até a frutificação, diminuindo ao final do ciclo; a TCRPA e a TALPA foram elevadas no início e depois decresceram. Ao ser analisado o efeito do tratamento mais salino $\left(\mathrm{T}_{4}\right)$, observa-se que, na TCAPA, este agiu de forma a retardar o crescimento, propiciando valores quase constantes de TCRPA e TALPA.

$\mathrm{Na}$ análise de variância do experimento II, os efeitos do fator tratamento foram não significativos $(p>0,05)$ para IAF, FSPA e RAFPA, e significativos a 0,01 de probabilidade para AFE; o fator DAS foi altamente significativo $(\mathrm{p}<0,01)$ em todas as características avaliadas, enquanto na interação de tratamentos versus DAS apenas a AFE foi altamente significativa. O aumento da salinidade da água, exceto para FSPA, reduziu linearmente todas as características avaliadas, segundo as equações: IAF $\left(\mathrm{m}^{2} \mathrm{~m}^{-2}\right)=-0,0650 * \mathrm{CEa}+1,7637$ $\left(\mathrm{R}^{2}=0,69\right) ;$ FSPA $\left(\right.$ g planta $\left.^{-1}\right)=-5,7792^{\text {ns }}$ $\mathrm{CEa}+139,95\left(\mathrm{R}^{2}=0,73\right)$; $\operatorname{AFE}\left(\mathrm{cm}^{2} \mathrm{~g}^{-1}\right)=$ $-6,9546 * * \mathrm{CEa}+242,21\left(\mathrm{R}^{2}=0,96\right) \mathrm{e}$ RAFPA $\left(\mathrm{cm}^{2} \mathrm{~g}^{-1}\right)=-2,2023 * \mathrm{CEa}+$ $112,38\left(\mathrm{R}^{2}=0,79\right)$.

Para o desdobramento de DAS dentro de cada nível de salinidade, o IAF teve resposta cúbica nos três tratamentos mais salinos e linear para o menos salino (Figura 1), verificando-se, portanto, pelas equações, valores máximos aos 54,59 e 55 DAS e maior taxa de crescimento aos 33, 37 e 36 DAS, respectivamente, nos tratamentos $\mathrm{T}_{2}, \mathrm{~T}_{3}$ e $\mathrm{T}_{4}$. Em $\mathrm{T}_{1} \mathrm{o}$ efeito linear aconteceu, provavelmente, por uma retomada natural de crescimento da cultura que, após diminuir de $2,52 \mathrm{~m}^{2} \mathrm{~m}^{-2}$ para $2,45 \mathrm{~m}^{2} \mathrm{~m}^{-2} \mathrm{da}$ 
antipenultima coleta (51 DAS) para a penúltima (58 DAS), voltou a aumentar para $3,11 \mathrm{~m}^{2} \mathrm{~m}^{-2}$ na última coleta $(64$ DAS). Em $\mathrm{T}_{3}$, embora tenha ocorrido o mesmo comportamento verificado para $\mathrm{T}_{1}$, devido ao aumento da última coleta ter sido proporcionalmente inferir ao de $\mathrm{T}_{1}$, a equação de melhor ajuste foi a cúbica. $\mathrm{Em} \mathrm{T}_{4}$ verifica-se que, em todas as coletas, os valores de IAF são inferiores aos dos demais tratamentos e sempre crescentes até a penúltima coleta, com leve queda no final, indicando o mesmo retardamento do crescimento ocorrido no experimento I (Figura 1).

Enfatiza-se o fato de que, para acelerar e uniformizar o processo de maturação e incrementar o teor de açúcar dos frutos, um dia antes de cada colheita, se provoca estresse hídrico pela diminuição da lâmina de irrigação aplicada. A não utilização de defensivos agrícolas e de irrigação, antecedendo o início da colheita, diminui o crescimento da planta, antecipando o seu ciclo natural, que poderia se estender por mais dias, criando novas folhas e produzindo mais frutos, embora fossem de qualidade inferior. Do exposto, certifica-se de que o declínio natural esperado para IAF no final do ciclo e, ao ser modificado por fatores, como o teor de umidade do solo, a infestação por pragas e doenças e o grau de precocidade das plantas, possa inexistir ou aparecer de forma tão sutil, que nos dois experimentos os quatro tratamentos estudados apresentaram ajuste ao modelo linear, com significância a 0,01 de probabilidade, não sendo os escolhidos por serem de menor grau que o cúbico, que apresenta significância na maioria dos tratamentos estudados, além de representar melhor esta característica ao longo dos DAS. A verificação de ataque menos intenso da mosca-branca (Bemísia argentifólia), minador (Liriomyza sp) e pulgão (Aphis gossypii) no experimento II que no I, pode ter contribuído para maior IAF no experimento II (Tabela 2), ao longo do ciclo, em todos os tratamentos estudados. Verificou-se, também, que a salinidade média do solo durante o ciclo da cultura foi maior no experimento I que no II (Tabela 3), contribuindo para que no segundo experimento o IAF fosse maior.

Tabela 2. Valores médios de índice de área foliar (IAF), fitomassa seca da parte aérea (FSPA), área foliar específica (AFE) e razão de área foliar da parte aérea (RAFPA) durante o ciclo do meloeiro, irrigado com diferentes níveis de salinidade da água, nos experimentos I e II. Mossoró-RN, 2001 e 2002.

\begin{tabular}{|c|c|c|c|c|c|}
\hline Experimento & Tratamento & IAF $\left(m^{2} m^{-2}\right)$ & $\begin{array}{c}\text { FSPA } \\
\left(\text { g }_{\text {planta }}{ }^{-1}\right)\end{array}$ & $\begin{array}{c}\text { AFE } \\
\left(\mathrm{cm}^{2} \mathbf{g}^{-1}\right)\end{array}$ & $\begin{array}{l}\text { RAFPA } \\
\left(\mathrm{cm}^{2} \mathrm{~g}^{-1}\right)\end{array}$ \\
\hline \multirow{4}{*}{ I } & $T_{1}\left(0,6 d^{\prime} m^{-1}\right)$ & 1,05 & 70,31 & 238,25 & 128,40 \\
\hline & $\mathrm{T}_{2}\left(1,9 \mathrm{dS} \mathrm{m}^{-1}\right)$ & 0,91 & 69,37 & 226,80 & 123,57 \\
\hline & $\mathrm{T}_{3}\left(3,2 \mathrm{dS} \mathrm{m}^{-1}\right)$ & 0,91 & 61,09 & 214,55 & 123,28 \\
\hline & $\mathrm{T}_{4}\left(4,5 \mathrm{dS} \mathrm{m}^{-1}\right)$ & 0,65 & 55,56 & 204,61 & 113,35 \\
\hline \multirow{4}{*}{ II } & $T_{1}\left(0,6 d^{\prime} m^{-1}\right)$ & 1,66 & 130,84 & 240,07 & 109,25 \\
\hline & $\mathrm{T}_{2}\left(1,9 \mathrm{dS} \mathrm{m}^{-1}\right)$ & 1,71 & 136,21 & 226,95 & 110,77 \\
\hline & $\mathrm{T}_{3}\left(3,2 \mathrm{dS} \mathrm{m}^{-1}\right)$ & 1,61 & 123,93 & 217,93 & 105,63 \\
\hline & $\mathrm{T}_{4}\left(4,5 \mathrm{dS} \mathrm{m}^{-1}\right)$ & 1,41 & 109,89 & 212,94 & 101,42 \\
\hline \multirow{2}{*}{ I } & CV $1(\%)$ & 36,85 & 34,53 & 17,92 & 14,38 \\
\hline & CV $2(\%)$ & 42,35 & 41,44 & 13,31 & 14,41 \\
\hline \multirow{2}{*}{ II } & CV $1(\%)$ & 26,78 & 44,67 & 6,67 & 12,00 \\
\hline & CV $2(\%)$ & 32,79 & 39,23 & 9,19 & 13,12 \\
\hline
\end{tabular}

Tabela 3. Valores médios da condutividade elétrica do extrato de saturação do solo, em dS $\mathrm{m}^{-1}$, na camada de 0 a $0,45 \mathrm{~m}$, determinados nos tempos $0,30,50$ e 70 dias após a semeadura (DAS) dos experimentos I e II, para os tratamentos $\mathrm{T}_{1}$ a T 4 . Mossoró-RN, 2001 e 2002.

\begin{tabular}{|c|c|c|c|c|c|c|c|c|}
\hline \multirow{2}{*}{$\begin{array}{l}\text { Tempo } \\
\text { (DAS) }\end{array}$} & \multicolumn{4}{|c|}{ Experimento I } & \multicolumn{4}{|c|}{ Experimento II } \\
\hline & $T_{1}$ & $T_{2}$ & $T_{3}$ & $T_{4}$ & $T_{1}$ & $T_{2}$ & $T_{3}$ & $T_{4}$ \\
\hline 0 & 1,01 & 1,01 & 1,01 & 1,01 & 0,78 & 0,67 & 0,71 & 0,84 \\
\hline 30 & 2,54 & 3,76 & 5,74 & 7,51 & 1,95 & 2,28 & 3,72 & 4,82 \\
\hline 50 & 4,51 & 7,11 & 8,89 & 10,81 & 1,37 & 2,75 & 4,94 & 7,40 \\
\hline 70 & 2,90 & 5,20 & 6,97 & 10,06 & 0,97 & 3,20 & 4,40 & 6,13 \\
\hline
\end{tabular}

Na FSPA a resposta foi quadrática nos quatro tratamentos estudados, sendo que nos três primeiros as equações foram semelhantes e superiores à do tratamento $\mathrm{T}_{4}$ (Figura 1). Este comportamento quadrático diferente do que ocorreu nos três primeiros tratamentos do experimento I (cúbico), deve ter sido idêntico ao verificado no IAF, em virtude do maior ataque da mosca minadora no experimento I, reduzindo a fitomassa das folhas, com reflexo na translocação de assimilados para os frutos neste experimento, mesmo que no final do ciclo.

Para AFE ao longo do ciclo a resposta foi quadrática para os tratamentos $\mathrm{T}_{1}$ e $\mathrm{T}_{2}$ e cúbica para $\mathrm{T}_{3}$ e $\mathrm{T}_{4}$ (Figura 1). As equaçõos cúbicas ajustadas para $\mathrm{T}_{3}$ e $\mathrm{T}_{4}$ neste experimento, assemelharam-se à encontrada no experimento I para o tratamento $T_{1}$, com oscilações ao longo do ciclo e menores valores no final. Os valores de AFE, no ponto de máximo e no final (65 DAS) do experi- mento II, foram de 233,59 e 200,23 e de 226,11 e $166,90 \mathrm{~cm}^{2} \mathrm{~g}^{-1}$, respectivamente, para $\mathrm{T}_{3}$ e $\mathrm{T}_{4}$, indicando superioridade de $\mathrm{T}_{3}$, neste experimento, a $\mathrm{T}_{1}$ no experimento I. As equações quadráticas com concavidades para baixo, ajustadas para $\mathrm{T}_{1}$ e $\mathrm{T}_{2}$, apresentaram pontos de máximo, respectivamente, a 38 e 37 DAS, diminuindo seus valores até o final do ciclo da cultura. Em todo o período estudado, a AFE em $\mathrm{T}_{1}$, de forma idêntica ao que aconteceu com o IAF, foi sempre superior à de $\mathrm{T}_{4}$. De modo geral, embora os modelos ajustados por tratamentos, para cada um dos experimentos, tenham sido diferentes, os valores de AFE foram menores no final do ciclo da cultura e em todas as coletas de cada tratamento os valores do experimento II tenderam a ser superiores aos do experimento I.

Na RAFPA, o efeito foi cúbico para $\mathrm{T}_{1}$ e $\mathrm{T}_{2}$ e linear para $\mathrm{T}_{3}$ e $\mathrm{T}_{4}$, diferindo um pouco do encontrado para o experimento I, que apresentou efeito linear 
para todos os tratamentos, porém se deve ressaltar que as equações cúbicas ajustadas para $\mathrm{T}_{1}$ e $\mathrm{T}_{2}$, no experimento II, são decrescentes, de pequena amplitude (oscilação), apresentando pontos de máximo e mínimo, respectivamente de 168,6 e 31,2 e de 166,6 e $38,7 \mathrm{~cm}^{2} \mathrm{~g}^{-1}$ a 22 e 64 e a 23 e 69 DAS, muito próximos dos valores determinados para $\mathrm{T}_{3} \mathrm{e}$ $\mathrm{T}_{4}$ através de suas equações lineares decrescentes, neste mesmo período do ciclo da cultura (Figura 1).

O comportamento da TCAPA com os DAS para todos os tratamentos foi linear, indicando crescimento uniforme, com coeficientes angulares das equações de 0,$32 ; 0,35 ; 0,31$ e 0,21 para, respectivamente, $\mathrm{T}_{1}, \mathrm{~T}_{2}, \mathrm{~T}_{3}$ e $\mathrm{T}_{4}$ (Figura 2 ). $\mathrm{O}$ tratamento $\mathrm{T}_{4}\left(\mathrm{CEa}=4,5 \mathrm{dS} \mathrm{m} \mathrm{m}^{-1}\right)$, embora tendo apresentado coeficiente angular inferior aos dos demais, ainda suplantou o encontrado no experimento I, igual a 0,14 .

No experimento II, a TCRPA tendeu a cair ao longo do ciclo nos quatro tratamentos, de forma similar ao experimento I (Figura 2). Aguiar Netto et al. (2000) estudando a batata sob condições de estresse hídrico, e Urchei et al. (2000) e Rocha (2001) estudando o feijão, respectivamente sob condições de irrigação e sequeiro, verificaram, para estas culturas, a mesma tendência de queda da taxa de crescimento relativo. Apesar de Radford (1967) argumentar ser este índice fisiológico mais apropriado para se comparar efeitos de diferentes manejos agronômicos, por ser relativo e não depender de pressuposições matemáticas, verificou-se pouca diferença na TCRPA entre tratamentos no experimento II, no início do período analisado, com os tratamentos $\mathrm{T}_{1}, \mathrm{~T}_{3}$ e $\mathrm{T}_{4}$ assumindo valores muito próximos entre si e superiores a $\mathrm{T}_{2}$ e, a partir de 30 DAS verificou-se valores muito próximos para todos os tratamentos.

No tratamento menos salino $\left(\mathrm{T}_{1}\right)$ a TALPA foi quase constante ao longo do ciclo da cultura; enquanto nos tratamentos mais salinos, foi mais elevada no início, apresentando valores superiores aos tratamentos menos salinos, que depois decresceram e se equipararam aos 30 DAS, permanecendo praticamente constantes e iguais ao longo do ciclo, voltando a se elevar levemente apenas no final, com $\mathrm{T}_{2}$ suplantando os demais tratamentos. Este índice reflete a fotossíntese líquida (Benincasa, 1988) e tende a decrescer no final do ciclo das espécies vegetais, em razão da senescência foliar como verificaram Oliveira (1995), Gomes et al. (2000) e Nóbrega et al. (2001); entretanto, o aparecimento de drenos fortes, no final do ciclo, tende a aumentar a TALPA, conforme explica Negreiros (1995) e verificado por Aguiar Netto et al. (2000).

Observa-se para os dois experimentos, uma tendência de menor TCAPAem $\mathrm{T}_{4}$, que nos outros tratamentos menos salinos; uma tendência de queda da TCRPA em todos os tratamentos e, ainda que, embora a TALPA tenha apresentado tendência geral de queda, os tratamentos $\mathrm{T}_{4}$ e $\mathrm{T}_{1}$ apresentaram valores quase constantes nos experimentos I e II, respectivamente.

Pode-se concluir que os níveis de salinidade da água de irrigação afetaram negativamente o índice de área foliar (IAF), a fitomassa seca da parte aérea (FSPA), a área foliar específica (AFE) e a razão de área foliar da parte aérea (RAFPA), inibindo o crescimento, com maior intensidade no IAF e menor na RAFPA.

A taxa de crescimento absoluto da parte aérea (TCAPA) foi afetada pelo nível de salinidade da água de irrigação, enquanto a taxa de crescimento relativo da parte aérea (TCRPA) e a taxa de assimilação líquida da parte aérea (TALPA) não apresentaram nitidamente efeito dos sais ao longo do ciclo do meloeiro.

\section{REFERÊNCIAS}

AGUIAR NETTO AO; RODRIGUES JD; PINHO SZ. 2000. Análise de crescimento na cultura da batata submetida a diferentes lâminas de irrigação. Pesquisa Agropecuária Brasileira 35: 901-907.

ALLEN RG; PEREIRA LS; RAES D; SMITH M. 1998. Crop evapotranspiration: guidelines for computing crop water requirements. Rome: FAO. 300p. (FAO: Irrigation and Drainage Paper, 56)

ANASTASIO G; PALOMARES G; NUEZ F; CATALA MS; COSTA J. 1987. Salt tolerance among Spanish cultivars of Cucumis melo. Cucurbit Genetics Cooperative. p. 41-42. (Report, 10).
AWAD M; CASTRO PRC. 1983. Introdução à fisiologia vegetal. São Paulo: Nobel. 177p.

BENINCASA MMP.1988. Análise de crescimento de plantas. Jaboticabal: FUNEP. 42p.

COSTA MC. 1999. Efeitos de diferentes lâminas de água com dois níveis de salinidade na cultura do meloeiro. Botucatu: UNESP, 115p. (Tese).

EMBRAPA. Centro Nacional de Pesquisas de Solos. Sistema brasileiro de classificação de solos. Rio de Janeiro 1999. 412p.

FRANCOIS LE; MAAS EV. 1993. Crop response and management on salt-affected soils. In: PESSARAKALI M (edS). Handbook of plant and crop stress. New York: Marcel Dekker Inc. p. 149-181.

GOMES AA; ARAUJO AP; ROSSIELLO ROP; PIMENTEL C. 2000. Acumulação de biomassa, características fisiológicas e rendimento de grãos em cultivares de feijão irrigado e sob sequeiro. Pesquisa Agropecuária Brasileira 35: 1927-1937.

GOMES FP. 1987. Curso de estatística experimental. 12. ed. São Paulo: Nobel. 467p.

IBGE-INSTITUTO BRASILEIRO DE GEOGRAFIA E ESTATÍSTICA. 2005, 01 de julho. Sistema de recuperação automática - Sidra: Produção agrícola municipal. Quantidade produzida, valor da produção, área plantada, área colhida da lavoura temporária e produtividade. Disponível em http:// www.sidra.ibge.gov.br.

LIMA AA. 2001. Absorção e eficiência de utilização de nutrientes por híbridos de melão (Cucumis melo, L). Fortaleza: UFC. 60p. (Dissertação)

MAAS EV. 1990. Crop salt tolerance. In: TANJI KK (edS). Agricultural salinity assessment and management. New York: American Society of Civil Engineers. p. 262-304.

MACHADO EC; PEREIRA AR; FAHL JI; SILVA WJ;TEIXEIRA JPF. 1982. Análise quantitativa de crescimento de quatro variedades de milho em três densidades de plantio, através de funções matemáticas ajustadas. Pesquisa Agropecuária Brasileira 17: 825-833.

MARTINS LH. 1993. Avaliação da qualidade da água nos mananciais superficiais disponíveis para irrigação na zona Oeste Potiguar. Mossoró: ESAM. 97p. (Monografia).

MEDEIROS JF. 1992. Qualidade da água de irrigação e evolução da salinidade nas propriedades assistidas pelo "GAT" nos Estados do $R N, P B$ e CE. Campina Grande: UFPB. 173p. (Dissertação).

NEGREIROS MZ. 1995. Crescimento, partição de matéria seca, produção e acúmulo de macronutrientes de plantas de pimentão (Capsicum annuum L.) em cultivo podado e com cobertura morta. Viçosa: UFV. 187p. (Tese).

NERSON H; PARIS HS. 1984. Effects of salinity on germination, seedling growth, and yield of melons. Irrigation Science 5: 265-273.

NÓBREGA JQ; RAO TVR; BELTRÃO NEM; FIDELIS FILHO J. 2001. Análise de crescimento do feijoeiro submetido a quatro níveis de umidade do solo. Revista Brasileira de Engenharia Agrícola e Ambiental 5: 437-43. 
OLIVEIRA AD. 1995. Análise de crescimento e desenvolvimento da cultura de melão (Cucumis melo L.), em condições diferenciadas de lâminas de irrigação. Mossoró: ESAM. 83p. (Monografia).

OLIVEIRA M; MAIA CE. 1998. Qualidade físico-química da água para irrigação em diferentes aquíf́eros na área sedimentar do Estado do Rio Grande do Norte. Revista Brasileira de Engenharia Agrícola e Ambiental 2: 42-46.

PIZARRO FC. 1990. Riegos localizados de alta frenquencia: goteo, microaspersion, exudacion. 2.ed. Madrid: Mundi Prensa. 459p.
PORTO FILHO FQ; ALENCAR RD; MEDEIROS JF; HOLANDA JS; FERREIRA NETO M; ROCHA DGF; PORTO VCN. 2001. Avaliação de duas cultivares de melão amarelo (Golde Mine e AF646) submetidos a diferentes níveis de salinidade da água de irrigação. In: CONGRESSO BRASILEIRO DE ENGENHARIAAGRÍCOLA, 30. Anais... Foz do Iguassu: SBEA (CD ROM).

RADFORD PJ. 1967. Growth analysis formulae - their use and abuse. Crop Science 7: 171175 .
RICHARDS FJ. 1969. The quantitative analysis of growth. In: STEWARD, F.C. (ed). Plant physiology: A treatise. New York: Academic Press. p. 3-76.

ROCHA EL. 2001. Crescimento do feijão-de-corda em consórcio com milho. Mossoró: Mossoró. 52p. (Dissertação).

URCHEI MA; RODRIGUES JD; STONE LF 2000. Análise de crescimento de duas cultivares de feijoeiro sob irrigação, em plantio direto e preparo convencional. Pesquisa Agropecuária Brasileira 35: 497-506.

VIVANCOS AD. 1996. Fertirrigacion. 2. ed. Madri: Mundi-Prensa. 233p. 